\title{
Nueva guía de la Organización Mundial de la Salud para el tratamiento farmacológico de la hipertensión arterial en adultos
}

\author{
New guideline from the World Health Organization for the pharmacological treatment of hypertension in adults
}

\section{Comentado de:}

Guideline for the pharmacological treatment of hypertension in adults. Geneva: World Health Organization; 2021. Licence: CC BY-NC-SA 3.0 IGO. PMID: $34495610^{1}$

\section{Introducción}

Esta guía, recientemente publicada por la Organización Mundial de la Salud (OMS) para el tratamiento farmacológico de la hipertensión arterial (HTA) en la población adulta ${ }^{1}$, proporciona los lineamientos para el uso de agentes antihipertensivos basa- dos en evidencia relevante y actualizada, con relevancia particular para países de ingresos medios y bajos. Tanto la valoración de la confianza de la evidencia como la fuerza de las recomendaciones fueron desarrolladas de manera rigurosa bajo el enfoque GRADE.

\section{Resumen de las recomendaciones}

La Tabla 1 resume las recomendaciones principales. Las mismas están dirigidas a personas adultas no embarazadas en quienes se haya diagnosticado HTA, y que hayan sido asesoradas de manera adecuada sobre las modificaciones en el estilo de vida.

Tabla 1. Recomendaciones clave de la nueva guía para el tratamiento farmacológico de la HTA de la OMS. Notas: ARA: bloqueantes de los receptores de angiotensina; BCC: bloqueantes de los canales de calcio; ECV: enfermedad cardiovascular; HTA: hipertensión arterial; IECA: inhibidores de la enzima convertidora de angiotensina; TAS: presión arterial sistólica; TAD: presión arterial diastólica.

\begin{tabular}{|l|l|c|}
\hline \multicolumn{2}{|l|}{ Recomendación clave } & $\begin{array}{l}\text { Fuerza de recomendación } \\
\text { (confianza en la evidencia) }\end{array}$ \\
\hline $\begin{array}{l}\text { Inicio del } \\
\text { tratamiento } \\
\text { farmacologico } \\
\text { antihipertensivo }\end{array}$ & En personas con diagnóstico confirmado de HTA y TAS $\geq 140 \mathrm{~mm} \mathrm{Hg} \mathrm{o} \mathrm{PAD} \geq 90 \mathrm{~mm} \mathrm{Hg}$ & Fuerte (moderada a alta) \\
\cline { 2 - 3 } & En las personas con ECV y TAS entre 130 y $139 \mathrm{~mm} \mathrm{Hg}$ & Fuerte (moderada a alta) \\
\cline { 2 - 3 } & $\begin{array}{l}\text { En las personas sin ECV pero con alto riesgo cardiovascular, diabetes mellitus o enferme- } \\
\text { dad renal crónica y TAS entre } 130 \text { y } 139 \mathrm{~mm} \mathrm{Hg}\end{array}$ & Condicional (moderada a alta) \\
\hline $\begin{array}{l}\text { Tratamiento } \\
\text { inicial }\end{array}$ & Elegir entre: tiazidas y agentes similares a las tiazidas; IECA / ARA; BCC & Fuerte (alta) \\
\cline { 2 - 3 } & $\begin{array}{l}\text { Terapia combinada, preferiblemente en una sola píldora (para mejorar la adherencia y per- } \\
\text { sistencia), eligiendo entre: diuréticos (tiazidas), IECA / ARA y BCC }\end{array}$ & Condicional (moderada) \\
\hline $\begin{array}{l}\text { Objetivo } \\
\text { terapéutico }\end{array}$ & Tensión arterial <140/90 mm Hg en todos los pacientes con HTA sin comorbilidades & Fuerte (moderada) \\
\cline { 2 - 3 } & TAS $<130 \mathrm{mmHg}$ en pacientes con HTA y ECV conocida & Fuerte (moderada) \\
\cline { 2 - 3 } & $\begin{array}{l}\text { TAS }<130 \mathrm{~mm} \mathrm{Hg} \text { en las personas con alto riesgo cardiovascular e HTA (aquellos con alto } \\
\text { riesgo de ECV, diabetes mellitus y/o enfermedad renal crónica) }\end{array}$ & Condicional (baja) \\
\hline $\begin{array}{l}\text { Frecuencia del } \\
\text { seguimiento }\end{array}$ & Mensual al iniciar o cambiar la medicación antihipertensiva, hasta alcanzar el objetivo & Condicional (baja) \\
\cline { 2 - 3 } & Cada tres a seis meses para los pacientes cuya tensión arterial se encuentra bajo control & \\
\hline
\end{tabular}

\section{Comentario}

La guía resumida se focaliza en el tratamiento farmacológico de la HTA crónica en personas adultas correctamente diagnosticadas, no embarazadas, y previamente asesoradas sobre las medidas no farmacológicas de manejo de la HTA. Tras aplicar la herramienta AGREE de valoración crítica para este tipo de documentos ${ }^{2}$, consideramos que presenta una excelente calidad, con limitaciones menores en su desarrollo y reporte (verTabla 2).

Hasta hace pocos años era habitual la recomendación de iniciar el tratamiento de la HTA únicamente con medidas no farmacológicas, excepto en casos selectos (p. ej., pacientes con alto riesgo cardiovascular global [RCG]), Sin embargo, la mayoría de los pacientes no podrá alcanzar y mantener las metas de HTA a largo plazo sin utilizar tratamiento farmacológico, incluso los pacientes jóvenes con HTA leve y sin daño de órgano blanco. Algunas guías, como la del Ministerio de Salud de la Nación de Argentina ${ }^{3,4}$ y la del Instituto Nacional de Salud y Excelencia Clínica (NICE, por sus iniciales en inglés) del Reino Unido ${ }^{5}$, aún recomiendan diferir el inicio del tratamiento farmacológico en este último grupo de pacientes, haciendo especial hincapié en la toma de decisiones compartidas en el caso de la guía NICE. En cambio, la guía elaborada por la OMS ${ }^{1}$ propone la indicación del tratamiento farmacológico de manera temprana en la totalidad de las personas con diagnóstico de HTA. 
Tabla 2. Evaluación de la calidad metodológica de la guía para el tratamiento farmacológico de la hipertensión arterial de la Organización Mundial de la Salud. Fuente: elaboración propia.

\begin{tabular}{|l|l|}
\hline Dominio AGREE II & Evaluación cualitativa \\
\hline Alcance y objetivo & $\begin{array}{l}\text { Objetivo general y población diana claramente detallados. Descripción de los resultados esperados en relación con los } \\
\text { aspectos de salud abordados. }\end{array}$ \\
\hline $\begin{array}{l}\text { Participación de los } \\
\text { implicados }\end{array}$ & $\begin{array}{l}\text { Enumeración de los profesionales que intervinieron en la elaboración de la guía, su especialidad e institución, pero sin una } \\
\text { descripción del papel de cada uno en el grupo elaborador. Descripción de las estrategias utilizadas para la incorporación de } \\
\text { valores y preferencias de la población en las recomendaciones. Descripción clara de la audiencia primaria y secundaria. }\end{array}$ \\
\hline $\begin{array}{l}\text { Rigor en la elabora- } \\
\text { ción }\end{array}$ & $\begin{array}{l}\text { Descripción del proceso utilizado para reunir y sintetizar la evidencia -aunque sin detallar los criterios de elegibilidad- y } \\
\text { los métodos para formular las recomendaciones. Evaluación del cuerpo de la evidencia con su respectiva interpretación. } \\
\text { Consideración de los beneficios y los riesgos en la elaboración de las recomendaciones. Relación explíita entre cada } \\
\text { una de las recomendaciones y la evidencia en la que se basan. Incorporación de revisores externos, su propósito y las } \\
\text { afiliaciones de cada uno. Plazo definido para una próxima actualización. }\end{array}$ \\
\hline Claridad & Recomendaciones presentadas de manera clara, concisa y fácilmente identificables. \\
\hline Aplicabilidad & $\begin{array}{l}\text { Descripción de las potenciales barreras para su aplicación. Algoritmos terapéuticos para la implementación de las reco- } \\
\text { mendaciones. Consideración de la costo-efectividad de las recomendaciones según el ámbito de aplicación. Establece una } \\
\text { frecuencia para el seguimiento de los pacientes pero no detalla la evaluación o exámenes sugeridos. }\end{array}$ \\
\hline $\begin{array}{l}\text { Independencia edi- } \\
\text { torial }\end{array}$ & $\begin{array}{l}\text { Financiamiento por los Centros para el Control y Prevención de Enfermedades de Estados Unidos y la OMS. Conflictos de } \\
\text { intereses de los miembros elaboradores detallados en el anexo 2. }\end{array}$ \\
\hline
\end{tabular}

Otro aspecto novedoso en el cuidado de las personas con HTA que esta guía retoma es la indicación cada vez más frecuente de terapia farmacológica dual desde el inicio. Si bien de manera tradicional se recomendaba el inicio con monoterapia (salvo en casos excepcionales) para luego ir escalonando el tratamiento en el caso de no alcanzar las metas buscadas, en la actualidad la mayoría de las guías recomiendan la terapia con dos fármacos desde el inicio del tratamiento, mientras que reservan la monoterapia para ciertos casos especiales, como los pacientes jóvenes con HTA leve y bajo RCG ${ }^{6}$. En este sentido, la guía de la OMS, quizás en un intento de simplificar los algoritmos, va incluso más allá y propone el inicio farmacológico temprano con terapia combinada de todos los pacientes adultos ${ }^{1}$.

Respecto de las metas de presión arterial, si bien puede haber diferencias menores (de $5 \mathrm{mmHg}$ ) entre distintas guías, el consenso general es que aquellas personas con alto RCG tengan objetivos terapéuticos aún menores que 140/90, en general, con TAS deseada menor a $130 \mathrm{mmHg}^{1,6,7}$.

Por último, la guía de la OMS ${ }^{1}$ no considera a los betabloqueantes como drogas de primera línea en el tratamiento de la HTA, en consonancia con la mayoría de las guías actuales de tratamiento ${ }^{6,7}$, si bien aún se encuentran recomendados en la guía argentina ${ }^{3,4}$.

Una de las debilidades del documento resumido es que, si bien incluye algunas consideraciones respecto a los valores y preferencias de los pacientes en relación a las metas terapéuticas, este aspecto no es abordado en profundidad. La inclusión de lineamientos claros para la toma de decisiones compartidas como parte fundamental del tratamiento de las enfermedades crónicas representa un desafío en la mayoría de las guías de práctica clínica.

Cabe destacar, además, que la guía de la OMS presenta un breve apartado de HTA y COVID-19. En él establece que, según la evidencia disponible, el grupo elaborador no encontró asociación entre el uso de los inhibidores de la enzima convertidora de angiotensina o los bloqueantes de los receptores de angiotensina y una mayor severidad de la enfermedad producida por el virus SARS-CoV-2.

\section{Conclusiones de las comentadoras}

La nueva guía para el tratamiento de la HTA desarrollada por la OMS provee una base para el abordaje farmacológico en los adultos diagnosticados con este factor de riesgo cardiovascular en las poblaciones de recursos medios y bajos. Considerando las diferencias mencionadas con otras guías, y evaluando desde las conductas más conservadoras a las más intervencionistas, parece razonable realizar un balance entre las mismas a la hora de definir las conductas clínicas: iniciar la terapia farmacológica en forma temprana (a los 3 a 6 meses si no se alcanzan las metas con las medidas no farmacológicas en pacientes jóvenes con HTA leve y RCG bajo, antes en el resto de los pacientes), hacerlo con combinaciones de dos drogas en dosis medias (pudiendo indicar la monoterapia a adultos jóvenes con bajo RCG y HTA leve), y evitar los betabloqueantes como fármacos de primera línea.

Bárbara Valente [ Departamento de Salud, Carrera de Medicina, Universidad Nacional de La Matanza. valentebarbara@hotmail.com ]

Julieta Aldana Salas Apaza [ Departamento de Salud, Carrera de Medicina, Universidad Nacional de La Matanza. julietasalasapaza@gmail.com ]

María Victoria Salgado [ Unidad de Conocimiento Traslacional Hospitalaria Patagónica, Hospital SAMIC EI Calafate; Centro de Estudios de Estado y Sociedad; Departamento de Medicina, Instituto Universitario Hospital Italiano de Buenos Aires. victoria.salgado@cedes.org ]

Valente B, Salas Apaza JA, Salgado MV. Nueva guía de la Organización Mundial de la Salud para el tratamiento farmacológico de la hipertensión arterial 
en adultos. Evid Actual Pract Ambul. 2021;24(4):e002163. Available from: https://dx.doi.org/10.51987/EVIDENCIA.V25I1.6984. Comentado de: Guideline for the pharmacological treatment of hypertension in adults. Geneva: World Health Organization; 2021. Licence: CC BY-NC-SA 3.0 IGO. PMID: 34495610

\section{Referencias}

1. World Health Organization. Guideline for the pharmacological treatment of hypertension in adults [Internet]. and others, editor. Geneva: World Health Organization; 2021. Available from: https:/www.ncbi.nlm.nih.gov/books/NBK573631/.

2. Brouwers MC, Kho ME, Browman GP, et al. AGREE II: advancing guideline development, reporting, and evaluation in health care. Prev Med [Internet]. 2010;51(5):421-424. Available from: 10.1016/j.ypmed.2010.08.005

3. Casetta B, Alcuaz MA, Bertarini F, et al.. Guía de Práctica Clínica Nacional sobre Prevención, Diagnóstico y Tratamiento de la Hipertensión Arterial.; 2019. Available from: https://bancos.salud.gob.ar/sites/default/files/2021-07/guia-de-practica-clinica-nacional-hipertension-arterial-2019-versionbreve.pdf.

4. Salas J, Valente B, Valverdi R, et al. Nueva guía de hipertensión arterial en Argentina. Evid actual pract ambul. 2020;22(4):e002038. Available from: 10.51987/evidencia.v22i4.4262; http://www.evidencia.org.ar/index.php/Evidencia/article/view/4262.

5. National Institutes for Health and Care Excellence. Hypertension in adults: diagnosis and management; 2019.Available from: https://www.nice.org. uk/guidance/ng136.

6. Williams B, Mancia G, Spiering W, M, et al. ESC/ESH Guidelines for the management of arterial hypertension. Eur Heart J. 2018;39(33):3021-3104. Available from: 10.1093/eurheartj/ehy339.

7. Whelton PK, Carey RM, Aronow WS, et al. ACC/AHA/AAPA/ABC/ACPM/AGS/APhA/ASH/ASPC/NMA/PCNA Guideline for the Prevention, Detection, Evaluation, and Management of High Blood Pressure in Adults: A Report of the American College of Cardiology/American Heart Association Task Force on Clinical Practice Guidelines. Hypertension. 2017;71(6):e13-e115. Available from: 0.1161/HYP.0000000000000065. 